\title{
Theoretical Basis and Experimental Means of the Hydrophilic Nanostructure Formation Process on the Titanium Surface by Direct Laser Irradiation
}

\author{
Sharif E. Guseynov ${ }^{1,2,3}$, Jekaterina V. Aleksejeva ${ }^{1}$, Ruslans Aleksejevs ${ }^{4}$, Uldis Zaimis ${ }^{1,2},{ }^{1}$ Liepaja \\ University, Institute of Fundamental Science and Innovative Technologies, ${ }^{2}$ Liepaja University, \\ Faculty of Science and Engineering, " "Entelgine" Research \& Advisory Company, Ltd., \\ ${ }^{4}$ Lomonosov Moscow State University, Faculty of Mechanics and Mathematics, Department of \\ Mathematics
}

\begin{abstract}
One of the most promising ways of periodic and/or nonperiodic nanostructure formation on implants' material surface, particularly on a titanium surface, providing the implants with the desired adhesive and wettability properties, is direct laser nanostructuring which uses only a laser beam with no atomic-force microscope's ancillary needle or masks in order to form surface nanorelief. In the present paper, the model of a nanostructure formation on a solid surface by microsecond laser pulses melting the material is described. It is shown that the typical size of the surface nanostructure formed depends on the laser wavelength, pulse energy, pulse repetition rate, and pulse duration. Within the present study a series of six experiments devoted to direct laser nanostructuring of titanium and copper surfaces was carried out. Besides, the effects of nanoroughness on the contact and sliding angles on hydrophilic surfaces were studied theoretically and experimentally.
\end{abstract}

Keywords: nanoroughness of surface, contact angle, nanostructured hydrophilic surface, laser irradiation

\section{Introduction}

At the present time nanostructures are given additional attention for being an extremely promising object regarding its application in different fields of science, technology and medicine. Surface nanostructuring leads to the improvement of nanotribological [1], thermalphysic and thermodynamic [2], electron-emission [3] properties of materials, promotes the increase of implants' biocompatibility ([4], [5], [6]) as well as leads to the desired alteration of adhesive properties ([7], [8], [9]) and wettability ([10], [11]). One of the most promising ways of periodic and/or nonperiodic nanostructure formation on implants' material surface, particularly on a titanium surface, providing the implants with the desired adhesive and wettability properties, is direct laser nanostructuring (see [12], [13] and respective references given in these) which uses only a laser beam with no atomic-force microscope's ancillary needle or masks in order to form a surface nanorelief. The advantage of this method consists in its simplicity and flexibility: (a) the use of a single laser beam of a small size allows one to achieve a high locality of exposure corresponding to the size (100x100 micrometer) of a separate laser spot; (b) the use of a softwareprogrammable laser beam scanning on the implants' material surface with a high irradiation pulse repetition frequency allows to nanostructure sufficiently large surface areas within almost arbitrary boundaries with high spatial resolution.

This study, which to a certain extent is a continuation of the research [14], is devoted to the formation of a hydrophilic nanostructure on the surface of a ball-shaped titanium head of a human hip joint endoprosthesis using direct laser nanostructuring. The necessity of hydrophilicity property of the implant surface is due to the fact that the state of the surface of titanium implants plays a decisive role in cell-cell adhesion, because the interaction between a biological environment (hard and soft tissues, blood, body fluids and serum, etc.) and the implant occurs on its surface, and the biological response from the living tissue depends on a variety of surface properties, such as chemical composition, purity, texture and topography, surface energy, corrosion resistance, and the tendency to denaturalization of the surrounding proteins. In this work, following the work [15], we will consider that a partially hydrophilic or hydrophobic surface with respect to any fluid is such a solid surface that for it the fluid gives a finite contact angle: if the contact angle $\theta$ between a drop of the liquid and the solid surface varies within the interval $\left(0, \frac{\pi}{2}\right)$, then this 
surface is called hydrophilic; if $\theta \in\left(\frac{\pi}{2}, \frac{5 \cdot \pi}{6}\right]$, then the surface is called hydrophobic; if $\theta \in\left(\frac{5 \cdot \pi}{6}, \pi\right]$, then the surface is classified as ultrahydrophobic; with $\theta=0$ and $\theta=\pi$ we have correspondingly complete wettability and complete nonwettability of the surface.

\section{Scientific basis: mathematical models and design formulas \\ Surface energy, surface tension and surface forces near the phase interfaces}

Within representation of continuous medium, while analyzing two-phase systems, phase boundaries are interpreted as geometric surfaces. However, real phase boundaries are thin transition layers of a rather complicated structure. Molecules which form the transition layer interact with the molecules of both adjacent phases, that is why the properties of a transition layer differ from the properties of a substance in the phases. This is the reason why during the interpretation of the phase boundaries some phenomenological properties appear. A correct description of microsurface forces lies in the foundation of surface tension's and capillarity's interpretation both in the old approach of Clairaut and Laplace and in the new approach of Bakker ([16], [17], [18]), which is based on introducing the pressure tensor in the interphase area. The description of microsurface forces should begin with the definition of interphase energy on the surface of the phase boundary of phases solid-liquid (and also of the phases of liquid-liquid, liquid-gas, solid-solid) and proceed with the definition of surface forces in the interphase area. It is known [19] that if both phases are fluid (liquid-liquid, liquid-gas), then their phase boundary can be stretched or it can shrink not affecting the thermodynamic equilibrium; if one phase is solid and the other is liquid or gaseous, then the surface of their phase boundary can experience only elastic deformations, however some degree of freedom appears if the liquid is able to slide on the sufficiently smooth solid material (for example, when mercury slides down a glass; water slides on a paraffin surface; blood or body fluids slide down a burnished surface of a titanium implant, etc.); if both phases are solid, then the behaviour of the phase boundary is similar to the case solid-liquid, however the implementation of a real phase contact is possible only with atomic smoothness or requires plastic deformation, or prior to melting, or evaporation and condensation with subsequent crystallization. Only in the case when both phases are fluid, a direct measurement of the phase boundary tension is possible. In the case, when both phases are solid, a direct measurement of the phase boundary tension is achievable with some approximation if and only if in some temperature region the yield point is lowered. In the case, when one phase is solid and the other is gaseous, a direct measurement of the phase boundary tension is impossible. The main mistakes made while measuring the surface energy in the process of the formation of a new surface are: (a) omission of an irreversible part of a fracture work, which turns into heat or energy residual deformations; (b) omission of the fact that fresh (that is, just created) surfaces are in nonequilibrium state with higher energy - they appear to be covered by charge with density of up to $3.5 \cdot 10^{-4} \mathrm{C} \cdot \mathrm{m}^{-2}$, that consumes part of the fracture work. The special state of fresh surfaces is expressed not only by increased energy, but also by a higher chemical activity. Relaxation down to an equilibrium state usually occurs for about ten minutes due to the electron emission and surface conduction.

Formation of a new phase boundary is connected with the consumption of work in order to move a part of molecules from the phases to the surface layer. In isothermal conditions the work of formation of the phase surface's element $d F$ is equal to the increment of Helmholtz energy $d U_{F}$ of the surface: $d U_{F}=\sigma \cdot d F$, where $\sigma$ is the specific Helmholtz energy of the phase boundary, which decreases as the temperature increases and at critical temperature $T_{c . t}$ achieves the approximate value of zero (in steam-andliquid systems $\sigma=0$, and the difference between the phases disappears). The values of $\sigma$ for the surface of a solid body on a border with a liquid are not identical to the corresponding coefficients of surface tension ([20]). Accurate theoretical formulas for the calculation of liquids' surface tension coefficient do not exist. The main methodological difficulty of calculation of the surface tension is the problem of ensuring solid surface purity, when it is in contact with a liquid, as well as the purity of the liquid. There are some methods of experimental calculation among which the most popular are [21]: the maximum gas bubble pressure method; capillary rise method; method of ring lifting; electromagnetic measurement methods; the drop weight method; hanging drop method; lying bubble or drop method. The simplest experimental method of determining the empirical dependence of the phase boundary's specific 
Helmholtz energy on the temperature is the following relation obtained from the theory of corresponding

states ([22]): $\sigma=\sigma_{0} \cdot\left(1-\frac{T}{T_{\text {c.t. }}}\right)^{\frac{11}{9}}, \quad$ where $\sigma_{0}$ is determined by the experiments carried out with one constant value of temperature. This formula has shown itself well working for liquids with homonuclear molecules, however it can be approximatively used also for the majority of other liquids. For calculations of water's $\sigma$ it is recommended to use the following interpolation formula: $\sigma=0.235\left(1-\frac{T}{T_{\text {c.t. }}}\right)^{1.256}\left(1-0.625 \frac{T_{c . t .}-T}{T_{\text {c.t. }}}\right)$.

\section{Wetting process}

In the introduction we mentioned (following the work [15] the contact angle of wetting $\theta$ between a solid surface and a tangent to the phase boundary gasliquid. From the minimality condition of the surface's Helmholtz energy ([23], [24]) one can obtain the following relation known as Young's law: $\theta=\operatorname{arccosine}\left(\frac{\sigma_{\text {solid, gas }}-\sigma_{\text {solid,liquid }}}{\sigma}\right)$. It is important to note that the contact angle is very sensitive to such difficult-to-control factors as roughness of a solid surface or presence of foreign impurity on the solid surface or in the liquid, particularly if this impurity is surface-active, which, concentrating on the thermodynamic phase boundary, cause surface tension lowering: for instance, $\mathrm{R}-\mathrm{OH}$ alcohols; R$\mathrm{COH}$ aldehydes; R-COOH carboxylic acids; $\mathrm{CH} 3-$, $\mathrm{C} 2 \mathrm{H} 5-, \mathrm{C} 17 \mathrm{H} 35-$, etc. hydrocarbon radicals; $\mathrm{COH}$ and $\mathrm{COOH}$ functional groups; oleic acid $\mathrm{C} 17 \mathrm{H} 33 \mathrm{COOH}$; stearic acid sodium $\mathrm{C} 17 \mathrm{H} 35 \mathrm{COONa}$; etc. The increase in the roughness of the solid surface increases its wettability, that is, it decreases the value of $\theta$. For some solid bodies participating in the phase solid-liquid in a certain interval of temperature, a dependence of the contact angle $\theta$ on the temperature is held [25]. As it is shown in [25], almost always an increase in temperature on a hydrophilic surface leads to the improvement of wettability, that is, to diminution of the angle $\theta$, but on hydrophobic surfaces - to the worsening of wettability, that is, to the increase of the angle $\theta$. Also it is important to note (especially regarding the implants) that the contact angle of wetting $\theta$ depends also on hysteresis of wetting- on the direction of wetting a solid body surface by a liquid, that is, on the fact, whether the liquid is flowing on the surface or down from it.

The phase separation surface's equilibrium and axisymmetric equilibrium separation surfaces of phases

If the liquid rests relative to the coordinate system related to Earth, then in hydromechanics this rest is called absolute rest; if the liquid rests relative to the coordinate system which moves with uniform acceleration relative to Earth, then this rest is called relative rest. For both absolute rest and relative rest Euler's equations in the vector form hold: $\vec{F}-\frac{1}{\rho} \cdot \nabla p=0$, where $\vec{F}$ is mass forces density vector, which, in the case of relative rest, includes inertial forces; $\rho$ is liquid density; $p$ is liquid pressure. Due to the fact that mass forces have potential in the majority of cases, one can write $\vec{F}=-\nabla \Phi$, where $\Phi$ is a force function. Consequently, Euler's equation turns into equation $\nabla \Phi+\frac{1}{\rho} \cdot \nabla p=0$, whose general integral for the cases, when $\rho=\rho(p)$, is the formula $\Phi+\Upsilon=$ const, called the equation of hydrostatics, where $\Upsilon=\int \frac{d p}{\rho}$ denotes pressure function. It should be noted that, if the liquid is a heavy compressible liquid, then out of all mass forces only the gravitational force affects it, so the general integral looks like $\Phi=g \cdot z+$ const, where $z$ denotes the coordinate which is counted vertically up. For a heavy compressible liquid the general integral of Euler's equation has the form $z+\frac{p}{g \cdot \rho}=$ const, and this formula represents hydrostatic distribution of pressure. When a gasliquid system is in the equilibrium state, then in each contacting phase, firstly, the equations of hydrostatics hold and, secondly, for every point on the phase boundary defined by a position vector $\vec{r}$ Laplace formula $p_{1}-p_{2}=2 \cdot \sigma \cdot H(\vec{r})$ is true, which means that on the boundary between two fluid phases the surface tension causes a pressure jump in the contacting phases, which is proportional to the average curvature $H$ of the phase boundary. From these two relations (equation of hydrostatics and Laplace formula) one can deduce the main differential equation of hydrostatic equilibrium, whose general integral $2 \cdot \sigma \cdot H(\vec{r})=\left(\rho_{\text {liquid }}-\rho_{\text {gas }}\right) \cdot \Phi(\vec{r})+$ const determines 
the shape of the phase boundary. In the most practically important axial-symmetric problems on liquid there are:a homogeneous gravitational field with intensity $g$, directed along the vertical axis $O Z$; centrifugal force field caused by a uniform rotation of the gas-liquid system around the same axis $O Z$ with an angular velocity $\omega$. In this case, the potential of mass forces has the form $\Phi=g \cdot z-\frac{1}{2} \cdot \omega^{2} \cdot d^{2}$, where $d=\sqrt{x^{2}+y^{2}}$ is the distance to the rotation axis. When the system rests, the potential of mass forces is determined by the formula $\Phi=g \cdot z$, but the main equation of hydrostatic equilibrium has the expression:

$2 \cdot \sigma \cdot H(z)=\delta \cdot g \cdot\left(\rho_{\text {liquid }}-\rho_{\text {gas }}\right) \cdot z+2 \cdot \sigma \cdot H(0)$,

where the term $2 \cdot \sigma \cdot H(0)$ represents the pressure jump on some "zero" level; $\delta=+1$ in the case, when the vertical axis $O Z$ is pointed up (and the gravitational field is pointed down), and $\delta=-1$ in the case, when the vertical axis $O Z$ is pointed down. For a resting system with a characteristic size $L_{c h . d}$. it is possible to use the main equation of hydrostatic equilibrium in order to obtain the scale of gravitational forces $f_{\text {grav }} \sim g \cdot\left(\rho_{\text {liquid }}-\rho_{\text {gas }}\right) \cdot L_{\text {ch.d. }}$ and the scale of surface tension forces $f_{\text {s.t. }} \sim \frac{\sigma}{L_{\text {ch.d. }}}$. Then Bond number Bo, which is the criterion of similarity in hydrodynamics and determines the relation between external forces (usually, gravity) and surface tension forces has the following form: Bo $=\frac{f_{\text {grav }}}{f_{\text {s.t. }}}=g \cdot\left(\rho_{\text {liquid }}-\rho_{\text {gas }}\right) \cdot \frac{L_{\text {ch.d. }}^{2}}{\sigma}$. The condition
$B o=1$ determines linear size of the region, where the gravitational forces and surface tension forces are equal: $L_{\text {lin.d. }}=\sqrt{\frac{\sigma}{g \cdot\left(\rho_{\text {liquid }}-\rho_{\text {gas }}\right)}}$. The quantity $L_{\text {lin.d. }}$ is called the capillary constant. For the majority of liquids with $p<<p_{\text {c.t. }}\left(p_{\text {c.t. }}\right.$ is critical pressure $)$, that is, on Earth conditions, the value of capillary constant varies within $1 \div 3 \mathrm{~mm}$. Obviously, if one divides the equation of hydrostatic equilibrium $2 \cdot \sigma \cdot H(z)=\delta \cdot g \cdot\left(\rho_{\text {liquid }}-\rho_{\text {gas }}\right) \cdot z+2 \cdot \sigma \cdot H(0)$ by the quantity $\sqrt{\sigma \cdot g \cdot\left(\rho_{\text {liquid }}-\rho_{\text {gas }}\right)}$, then it turns into the following dimensionless form: $2 \cdot \tilde{H}(\tilde{z})=\delta \cdot \tilde{z}+C$, where $\tilde{H}=L_{\text {lin.d. }} \cdot H, \quad \tilde{z}=\frac{z}{L_{\text {lin.d. }}}, C=2 \cdot H(0) \cdot L_{\text {lin.d. }}$.

\section{Melting process}

At sufficient energy density, duration and order of a laser pulse melting of solid body's surface occurs in our case, of a ball-shaped titanium head of a human hip joint endoprosthesis. When the laser pulse irradiation stops, the process of cooling the processed surface starts and is carried out by the means of heat removal deep into the solid phase and subsequent solidification of the surface layer. It is supposed that the power of laser irradiation is such that the phase transition solid-liquid occurs. Then, with appropriate approximation, temperature fields in the liquid $T_{\text {liquid }}(x, t)$ and solid $T_{\text {solid }}(x, t)$ phases can be described by the following boundary value problem ([13], [26], [27]):

$$
\begin{aligned}
& \frac{\partial T_{\text {liquid }}(x, t)}{\partial t}=a_{\text {liquid }} \cdot \frac{\partial^{2} T_{\text {liquid }}(x, t)}{\partial x^{2}}, 0<x<y(t), \\
& \frac{\partial T_{\text {solid }}(x, t)}{\partial t}=a_{\text {solid }} \cdot \frac{\partial^{2} T_{\text {solid }}(x, t)}{\partial x^{2}}, y(t)<x<\infty, \\
& \frac{\partial Q(t)}{\partial t}+\left.\lambda_{\text {liquid }} \cdot \frac{\partial T_{\text {liquid }}(x, t)}{\partial x}\right|_{x=0}=0, \\
& \left.T_{\text {liquid }}(x, t)\right|_{x=y(t)}=\left.T_{\text {solid }}(x, t)\right|_{x=y(t)}=T_{1},
\end{aligned}
$$




$$
\left.\lambda_{\text {liquid }} \cdot \frac{\partial T_{\text {liquid }}(x, t)}{\partial x}\right|_{x=y(t)}=H \cdot \rho \cdot y^{\prime}(t)+\left.\lambda_{\text {solid }} \cdot \frac{\partial T_{\text {solid }}(x, t)}{\partial x}\right|_{x=y(t)} .
$$

where $y(t)$ is the moving boundary of the phase transition; $Q(t)$ is the absorbed energy per surface unit for the time $t<<\tau$, here $\tau$ denotes the duration of a laser pulse; $a=\frac{\lambda}{c \cdot \rho}$ is thermal diffusivity, where $\lambda, \quad c$ and $\rho$ denote correspondingly thermal conductivity, specific heat and density of a metal

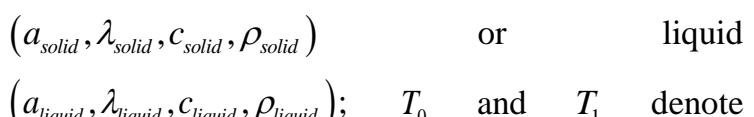

$$
\left.T_{\text {liquid }}(x, t)\right|_{x=0}=T_{2}=\left.T_{\text {liquid }}(x, \tau)\right|_{x=0}>T_{1},
$$

where $T_{2}$ is a temperature of molten metal on the Combining (1)-(3), (5) and (7) we can write that surface that can be defined by the heat balance equation. correspondingly the initial temperature of the solid body and the temperature of the phase transition; $H$ is the latent melting heat, which is absorbed on the moving phase boundary. Supposing that the temperature of the metal surface processed by laser pulse achieves phase transition point during the time $t_{0}<<\tau$ and the temperature of the liquid phase varies slightly during the further process $t_{0}<t<\tau$, we can replace the boundary condition (4) by the following more simple condition:

$$
\begin{aligned}
& \left.T_{2}=T_{1}+\frac{\operatorname{erf}\left(\frac{\beta}{\sqrt{2 \cdot a_{\text {liquid }}}}\right)}{2 \cdot c_{\text {liquid }} \cdot \rho \cdot \sqrt{a_{\text {liquid }}} \cdot\left(1-e^{-\frac{\beta^{2}}{2 \cdot a_{\text {liquid }}}}\right) \cdot \operatorname{erfc}\left(\frac{\beta}{\sqrt{2 \cdot a_{\text {solid }}}}\right)} \times, \quad-2 \cdot c_{\text {solid }} \cdot \rho_{\text {solid }} \cdot\left(T_{1}-T_{0}\right)\right\}, \\
& \times\left\{\left(\sqrt{\frac{\pi}{\tau}} \cdot Q(\tau)-\sqrt{2 \cdot \pi} \cdot H \cdot \rho_{\text {solid }} \cdot \beta\right) \cdot \operatorname{erfc}\left(\frac{\beta}{\sqrt{2 \cdot a_{\text {solid }}}}\right),\right.
\end{aligned}
$$

where $\operatorname{erf}(\omega)=\frac{2}{\sqrt{\pi}} \cdot \int_{0}^{\omega} e^{-\xi^{2}} d \xi$ is the Gauss error error function; the parameter $\beta$ can be found from function; $\operatorname{erfc}(\omega)=1-\operatorname{erf}(\omega)$ is the complementary

$$
\begin{gathered}
\frac{2 \cdot \sqrt{\frac{a}{\pi}} c \cdot \rho \cdot\left(T_{1}-T_{0}\right)}{\operatorname{erf} c\left(\frac{\beta}{\sqrt{2 \cdot a}}\right)}=\sqrt{2} \cdot H \cdot \rho \cdot \beta \cdot\left(e^{-\frac{\beta^{2}}{2 \cdot a}}-2\right)+\frac{Q(\tau)}{\tau} \\
Q(\tau)=H \cdot \rho \cdot y(\tau)+\int_{0}^{y(\tau)} c_{\text {solid }} \cdot \rho_{\text {solid }} \cdot\left(T_{1}-T_{0}\right) d x+\int_{0}^{y(\tau)} c_{\text {liquid }} \cdot \rho \cdot\left(T_{\text {liquid }}-T_{1}\right) d x+\int_{y(\tau)}^{\infty} c_{\text {solid }} \cdot \rho_{\text {solid }} \cdot\left(T_{\text {solid }}-T_{0}\right) d x
\end{gathered}
$$

The formulas (8)-(10) allow us to calculate the melting depth of different solid materials irradiated by laser pulses of various duration and energy. As it has already been mentioned above, under the influence of laser irradiation high temperature stress occurs in the metals. The deformation process in these conditions is followed by residual deformation, whose appearance is related to nonelastic effects and reorganization of crystal's defect structure. Stress' relaxation can have a heterogeneous character due to the formation of new structure zones called relaxation zones [32] in the old excited structure. Depending on 
the state of the system, on the conditions of external influence, on the degree of process development it may occur that relaxation zones can be centers of a new phase, groups of dislocations and disclinations, microcracks, clusters of atoms and vacancies forming clusters, micropore, dislocation loops, etc. One can imagine the deforming as three simultaneously coexistent phases: relaxation field determined by the

$$
\left\{\begin{array}{l}
\dot{\varphi}_{i, j}=-\kappa \cdot \varphi_{i, j}+g_{1} \cdot n \\
\dot{n}=-\gamma \cdot n+\frac{\varphi_{i, j} \cdot \sigma_{i, j}}{g_{2}} \\
\dot{\sigma}_{i, j}=v \cdot\left(\sigma_{i, k}-\sigma_{0}\right)-g_{3} \cdot \varphi_{i, j} \cdot n,
\end{array}\right.
$$

where parameters $\kappa, \gamma, v, g_{i}(i=\overline{1,3})$ are material constant; quantity $\sigma_{0}$ is determined by the applied external loads and corresponds to the residual stress set as the result of relaxation.

In the system (11) the term $-\kappa \cdot \varphi_{i, k}$ is the decay of the relaxation process; the term $-\gamma \cdot n$ describes disintegration of the formed relaxation zones; the term $v \cdot\left(\sigma_{i, k}-\sigma_{0}\right)$ describes the stress relaxation in linear approximation, when their interference is absent; the term $g_{1} \cdot n$ is related to the relaxation field generation due to the formation of relaxation zones; the term $\frac{\varphi_{i, k} \cdot \sigma_{i, k}}{g_{2}}$ takes into account the influence of parameter

$$
\varphi_{i, j}(r, t), \quad \text { stress field }
$$
corresponding to external load and relaxation zones with concentration $n(r, t)$. Temporary dependence $\varphi_{i, j}(r, t), \sigma_{i, j}(r, t)$ and $n(r, t)$ is defined by the following system of nonlinear differential equations:

$$
\varphi_{i, j}=D \cdot \Delta \varphi_{i, j}+\frac{g_{1}}{g_{2} \cdot \gamma} \cdot\left\{\left(\sigma_{0}-\frac{g_{2} \cdot \gamma}{g_{1}} \cdot \kappa\right) \cdot \varphi_{i, j}-\frac{g_{3} \cdot \sigma_{0}}{g_{2} \cdot \gamma \cdot v} \cdot \varphi_{i, j}^{3}\right\},
$$

where $D$ is the diffusion coefficient of atoms in a period can be calculated using the formula warmed-up zone.

If we take into account the diffusion's dispersion, then equation (12) will turn into the well-known Ginzburg-Landau generalized equation ([33], [34]). For $\sigma_{0}<\sigma_{c}=\frac{g_{2} \cdot \gamma}{g_{1}} \cdot \kappa$ equation (12) has one stable solution $\varphi_{i, j}(r, t)=0$; if exceeding of the critical value $\sigma_{0}>\sigma_{c}$ occurs, new coherent states of the $P=\frac{\sigma_{0}-\sigma_{c}}{2 \cdot \pi \cdot \sigma_{0}} \cdot \sqrt{\frac{3 \cdot D}{8 \cdot v}}$

Using the kinetic equation from the theory of surface nucleation ([2], [28], [29], [30]) as well as the variational principle for the main laws of thermal conduction, one can obtain ([31]) the following closed formula for the characteristic size-radius of a crystalline phase nucleus in a supercooled liquid:

$$
r(t)=v_{0} \cdot d \cdot e^{-\frac{U}{k \cdot T_{1}}} \cdot \frac{k \cdot T_{1}^{2}}{U \cdot \varepsilon} \cdot\left(\frac{h}{U+h}-e^{-\frac{U \cdot \varepsilon \cdot t}{k \cdot T_{1}^{2}}}+\frac{U}{U+h} \cdot e^{-\frac{(U+h) \cdot \varepsilon \cdot t}{k \cdot T_{1}^{2}}}\right)
$$


where $v_{0}$ is the Debye oscillation frequency of atoms in a supercooled liquid; $U$ is the activation energy for atom movement; $k \cdot T$ is thermal energy; $d$ is the characteristic size for a single atom; $h$ is the heat of the phase transformation for one atom; $\varepsilon$ is the average speed of melt cooling. Using the formula (13) one can precisely estimate the characteristic sizeradius of a crystalline phase nucleus, that is, the size of nanostructures appearing on the surface of the processing solid body, while it is melting under laser pulse irradiation.

\section{Experimental facilities, experiments and results Experimental facilities}

Within the present study we have carried out a series of six experiments devoted to direct laser nanostructuring of titanium (Ti) and copper $(\mathrm{Cu})$ surface. All the processed samples had the same sizes: $L \times W \times H=5 \times 3 \times 0.1 \mathrm{~mm}$. The surfaces of the samples had 14th surface finish class. The samples were placed in a laser facility chamber $\mathrm{Nd}^{3+}: \mathrm{YAG}$ crystal and were irradiated in a motionless laser beam. For a detailed analysis of the irradiated samples' profiles we used a desktop scanning electron microscope TM3030 with low vacuum regime, which allows us to carry out the experiments without sample preparation and to investigate samples without prior metal deposition; controlled by a simple and understandable interface with the functions of automatic focus, contrast and brightness setting; equipped with two detectors - of secondary and reflected electrons, - owing to which it is possible to get comprehensive information about the surface of the investigated samples. The laser facility $\mathrm{Nd}^{3+}$ :YAG crystal, which was used in order to carry out a series of experiments devoted to direct laser nanostructuring of surfaces of 4 titanium and 2 copper samples, has the following capabilities: laser wavelength: $532 \mathrm{~nm}$; maximum pulse energy: $0.15 \mathrm{~J}$; pulse repetition rate: $10 \mathrm{~Hz}$; initial pulse duration: $5 \div 9 \mathrm{~ns}$; pulse duration: $5 \div 400 \mu$ s. After repeated irradiation with one beam of microsecond $\mathrm{Nd}^{3+}: \mathrm{YAG}$ crystal-laser formation of nanosized structures on the copper and titanium surfaces was observed. Laser irradiation intensity was maximal in the center of the irradiation spot and was going down closer to its periphery. After repeated pulse action on one surface patch, a deep crater appeared in the spot's center. However, on the periphery, where the laser irradiation intensity was lower, one could observe only some surface melting. Using SEM-analysis (that is, using scanning electron microscopy with X-ray spectrometry microanalysis) no submicrometer structures were detected in the central high-intensity part of the spot. However, formation of nanorelief was detected in the peripheral part of the irradiation zone. For all six samples we detected roughness of a characteristic size along the surface on the surface in the peripheral low-intensity region of the spot as well as small-scale roughness in the form of saliences put over the surface. A round shape of the obtained nanoreliefs implies the surface being molten by the laser irradiation and subsequent crystallization with the formation of the above mentioned submicrometer structures. Taking into account the possibility of slight material melting, we can consider a surface under thermal flow of pulsed laser irradiation that provokes the solid-liquid phase transition.

\section{Experiment 1}

In this experiment copper foil was taken as a sample, and for its laser processing the following characteristics of microsecond laser facility $\mathrm{Nd}^{3+}:$ YAG crystal were chosen: laser wavelength $(\mathrm{LW}): 532 \mathrm{~nm}$; pulse energy (PE): $0.14 \mathrm{~J}$; pulse repetition rate (PRR): $10 \mathrm{~Hz}$; pulse duration $(\mathrm{PD})$ : $130 \mu$ s. The results of a single pulse action on this sample are shown in Fig.1. The obtained nanostructured surface has a good hydrophilic property with an average interfacial contact angle $\theta_{\text {average }} \approx 51^{\circ}$, where water was used as the liquid.

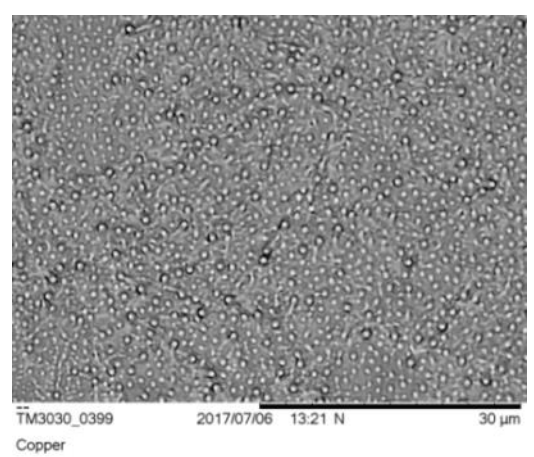

Fig. 1. Nanostructured copper surface (one-time processing) with an average contact angle of $51^{\circ}$ (good hydrophilic property) $\mathrm{Nd} 3+$ :YAG crystal-laser's features: $\mathrm{LW}=532 \mathrm{~nm}$; PE=0.14 J; $\mathrm{PRR}=10 \mathrm{~Hz} ; \mathrm{PD}=130 \mu \mathrm{s}$.

\section{Experiment 2}

In this experiment copper foil was again taken as a sample, and for its laser processing the following characteristics of the microsecond laser facility $\mathrm{Nd}^{3+}$ :YAG crystal were chosen: $\mathrm{LW}=532 \mathrm{~nm}$; $\mathrm{PE}=0.14 \mathrm{~J} ; \mathrm{PRR}=10 \mathrm{~Hz} ; \mathrm{PD}=130 \mu \mathrm{s}$. The results of a twofold pulse action on this sample are shown in 
Fig.2. The obtained nanostructured surface has a much worse hydrophilic property (more exactly, has a hydrophobic property) with an average interfacial contact angle $\theta_{\text {average }} \approx 115^{\circ}$, where water was used as the liquid.

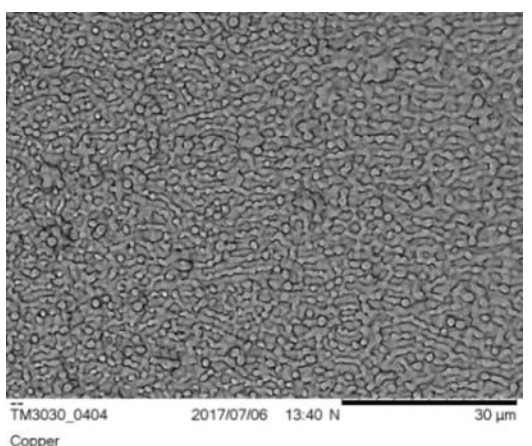

Fig. 2. Nanostructured copper surface (twofold processing) with an average contact angle of $115^{\circ}$ (hydrophobic property). $\mathrm{Nd} 3+$ :YAG crystal-laser's features: $\mathrm{LW}=532 \mathrm{~nm} ; \mathrm{PE}=0.14 \mathrm{~J}$; $\mathrm{PRR}=10 \mathrm{~Hz} ; \mathrm{PD}=130 \mu \mathrm{s}$.

\section{Experiment 3}

In this experiment titanium foil was taken as a sample, and for its laser processing the following characteristics of the microsecond laser facility $\mathrm{Nd}^{3+}$ :YAG crystal were chosen: $\mathrm{LW}=532 \mathrm{~nm}$; $\mathrm{PE}=0.15 \mathrm{~J} ; \mathrm{PRR}=10 \mathrm{~Hz} ; \mathrm{PD}=100 \mu \mathrm{s}$. The results of a single pulse action on this sample are shown in Fig.3. The obtained nanostructured surface has a very good hydrophilic property with an average interfacial contact angle $\theta_{\text {average }} \approx 28^{\circ}$, where both water and physiological solution $\mathrm{NaCl} 0.9 \%$ were used as the liquid.

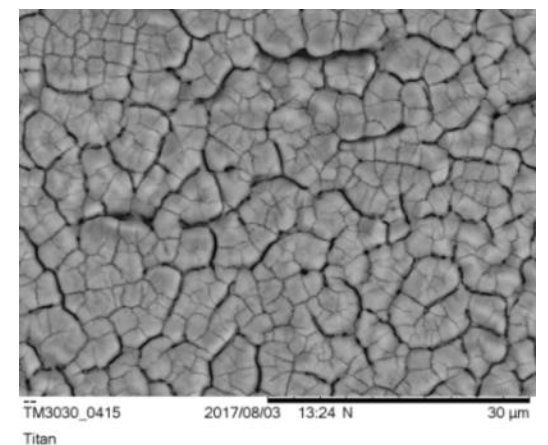

Fig. 3. Nanostructured titanium surface (one-time processing) with an average contact angle of $28^{\circ}$ (very good hydrophilic property). Nd3+:YAG crystal-laser's features: $L W=532 \mathrm{~nm}$; $\mathrm{PE}=0.15 \mathrm{~J} ; \mathrm{PRR}=10 \mathrm{~Hz} ; \mathrm{PD}=100 \mu \mathrm{s}$.

\section{Experiment 4}

In this experiment titanium foil was again taken as a sample, and for its laser processing the following characteristics of the microsecond laser facility
$\mathrm{Nd}^{3+}$ :YAG crystal were chosen $\mathrm{LW}=532 \mathrm{~nm}$; $\mathrm{PE}=0.15 \mathrm{~J} ; \mathrm{PRR}=10 \mathrm{~Hz} ; \mathrm{PD}=130 \mu \mathrm{s}$. The results of a single pulse action on this sample are shown in Fig.4. The obtained nanostructured surface has a good hydrophilic property with an average interfacial contact angle $\theta_{\text {average }} \approx 42^{\circ}$, however it is slightly worse than in the results of the third experiment. In this experiment both water and physiological solution $\mathrm{NaCl} 0.9 \%$ were used as the liquid.

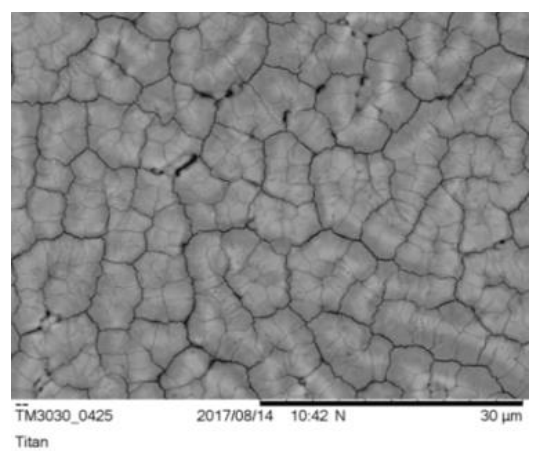

Fig. 4. Nanostructured titanium surface (one-time processing) with an average contact angle of $42^{\circ}$ (good hydrophilic property). $\mathrm{Nd} 3+$ :YAG crystal-laser's features: $\mathrm{LW}=532 \mathrm{~nm} ; \mathrm{PE}=0.15 \mathrm{~J}$; $\mathrm{PRR}=10 \mathrm{~Hz} ; \mathrm{PD}=130 \mu \mathrm{s}$.

\section{Experiment 5}

In this experiment titanium foil was again taken as a sample, and for its laser processing the following characteristics of the microsecond laser facility $\mathrm{Nd}^{3+}$ :YAG crystal were chosen: $\mathrm{LW}=532 \mathrm{~nm}$; $\mathrm{PE}=0.15 \mathrm{~J} ; \mathrm{PRR}=10 \mathrm{~Hz} ; \mathrm{PD}=85 \mu \mathrm{s}$. The processing was implemented by the stripes with the use of a lens for focusing of the laser beam. The results of a single pulse action on this sample are shown in Fig.5. The obtained nanostructured surface has the following hydrophilic property: the liquid (both water and physiological solution $\mathrm{NaCl} 0.9 \%$ ) flows only in one direction,

and $\theta_{\text {average }} \approx\left\{\begin{array}{l}66^{\circ} \text { on processed strips, } \\ 134^{\circ} \text { between processed strips. }\end{array}\right.$

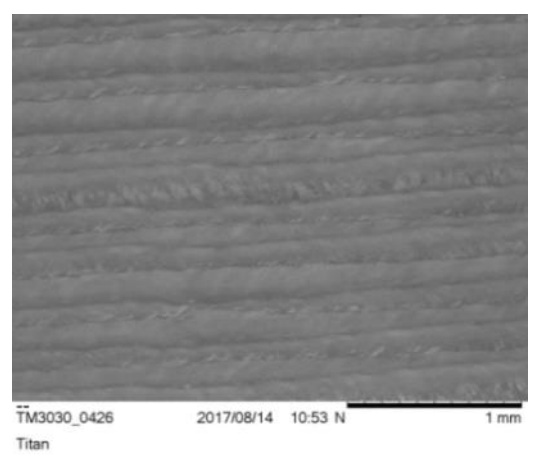

Fig. 5. Nanostructured titanium surface (one-time processing with a lens for focusing of a laser beam) with an average contact 
angle of $66^{\circ}$ on processed strips: the liquid flows only in one direction. Nd3+:YAG crystal-laser's features: $L W=532 \mathrm{~nm}$; $\mathrm{PE}=0.15 \mathrm{~J} ; \mathrm{PRR}=10 \mathrm{~Hz} ; \mathrm{PD}=85 \mu \mathrm{s}$.

\section{Experiment 6}

In this experiment titanium foil was again taken as a sample, and for its laser processing the following characteristics of the microsecond laser facility $\mathrm{Nd}^{3+}$ :YAG crystal were chosen: $\mathrm{LW}=532 \mathrm{~nm}$; $\mathrm{PE}=0.15 \mathrm{~J} ; \mathrm{PRR}=10 \mathrm{~Hz} ; \mathrm{PD}=300 \mu \mathrm{s}$. The results of a single pulse action on this sample are shown in Fig.6. The obtained nanostructured surface has a very good hydrophilic property with an average interfacial contact angle $\theta_{\text {average }} \approx 34^{\circ}$, where both water and physiological solution $\mathrm{NaCl} 0.9 \%$ were used as the liquid.

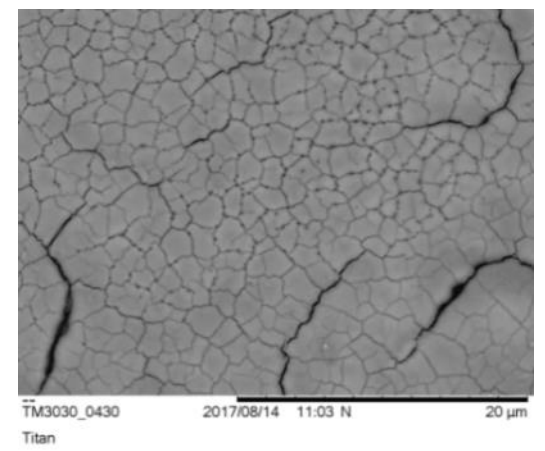

Fig. 6. Nanostructured titanium surface (one-time processing) with an average contact angle of $34^{\circ}$ (very good hydrophilic property). Nd3+:YAG crystal-laser's features: $L W=532 \mathrm{~nm}$; $\mathrm{PE}=0.15 \mathrm{~J} ; \mathrm{PRR}=10 \mathrm{~Hz} ; \mathrm{PD}=300 \mu \mathrm{s}$.

\section{Conclusions}

In this paper, the theoretical basis of a hydrophilic nanostructure formation process on a titanium surface by direct laser irradiation is described in detail. The design formulas, with the help of which it is possible to determine the shape of the phase interface; to calculate the melting depth of solid material irradiation with laser pulses of various duration and energy; to precisely estimate the characteristic sizeradius of a crystalline phase nucleus; etc., are given in closed form. Besides, in this paper, the experimental means and conditions, and the results of a series of six experiments carried out on direct laser nanostructuring of titanium and copper surfaces are described in detail.

\section{Acknowledgements}

For the first two co-authors, the present article was elaborated within the framework of the State Research Programme "Next Generation Information and Communication Technologies (NexIT)", Project No. 4.

\section{References}

1. Eletskii, A. V., Erkimbaev, A. O., Zitserman, V. Yu., Kobzev, G. A., Trakhtengerts, M. S. Thermophysical Properties of Nanoobjects: Data Classification and Validity Evaluation. Journal of High Temperature, 2012, Vol. 50, No. 4, p. 488-495.

2. Cao, G. Nanostructures and Nanomaterials: Synthesis, Properties, and Applications. London: Imperial College Press, 2004, xiv+433 p.

3. Bhushan, B., Israelachvili, J. N., Landman, U. Nanotribology: Friction, Wear and Lubrication at the Atomic Scale. Nature: International Journal of Science, 1995, Vol. 374, No. 6523, p. 607-616.

4. Hao, L., Lawrence, J. Laser Surface Treatment of Bio-Implant Materials. Chichester: John Wiley \& Sons, 2005, xix+211 p.

5. Oshida, Y. Bioscience and Bioengineering of Titanium Materials. Waltham: Elsevier, 2013, xiv+500 p.

6. Remeeva, E. A., Rozanova, I. B., Elinson, V. M., Sevastianov, V. I. Influence of Biomaterials Surface on Platelets Release ATP in vitro. International Journal of Artificial Organs, 2013, Vol. 26, No. 7, p. 634-639.

7. Israelachvili, J. N. Intermolecular and Surface Forces: With Applications to Colloidal and Biological Systems. New York: Academic Press, 1992, xxi+450 p.

8. Kendall, K. Adhesion: Molecules and Mechanics. Journal of Science, 1994, Vol. 263, No. 5154, p. 1720-1725.

9. Majumder, A., Ghatak, A., Sharma, A. Microfluidic Adhesion Induced by Subsurface Microstructures. Journal of Science, 2007, Vol. 318, No. 5848, p. 258-261.

10. Ferrara, M. C., Pilloni, L., Mazzarelli, S., Tapfer, L. Hydrophilic and Optical Properties of Nanostructured TItania Prepared by Solgel Dip Coating. Journal of Physics D: Applied Physics, 2010, Vol. 43, No. 9, 95301(37 p.).

11. Rios, P. F., Dodiuk, H., Kenig, S., McCarthy, S., Dotan, A. The Effects of Nanostructure and Composition on the Hydrophobic Properties of Solid Surfaces. Journal of Adhesion Science and Technology, 2006, Vol. 20, No. 6, p. 563-587.

12. Khomich, V. Yu., Urlichich, Yu. M., Shmakov, V. A., Tokarev, V. N., Galstyan, A. M., Mikolutskiy, S. I., Malinskiy, T. V., Ganin, D. V. Formation of Submicron Structures on the Surface of Zirconium Dioxide under Illumination of Nanosecond Laser. Journal of Inorganic Materials: Applied Research, 2013, Vol. 4, No. 3, p. 201-204.

13. Khasaya, R. R., Khomich, Y. V., Malinskiy, T. V., Mikolutskiy, S. I., Yamshchikov, V. A., Zheleznov, Y. A. Titanium Surface Processing by Nanosecond Laser Radiation. Letters on Materials, 2014, Vol. 4, No. 1, p. 45-48.

14. Zaimis, U., Guseynov, Sh. E. Scientifically Substantiated Guidelines for Physico-mathematical Modelling of Laser Surface-treatment of Wear-resistant Implants for Human Joint Replacements. In: Proceedings of the 11th International Scientific and Practical Conference "Environment. Technology. Resources", Vol. 3, 2017, p. 350-356.

15. Adamson, A. W., Gast, A. P. Physical Chemistry of Surfaces. New York: John Wiley \& Sons, 1997, xxi+784 p.

16. Современная теория капиллярности: к 100-летию теории капиллярности Гиббса / под редакцией А.И. Русанова, Ф.Ч. Гудрича. Ленинград: Химия, Ленинградское отделение, 1980, 340 р.

17. Blkerman, J. J. Capillarity before Laplace: Clairaut, Segner, Monge, Young. Archive for History of Exact Sciences, 1978, Vol. 18, No. 2, p. 103-122.

18. Bakker, G. Handbook on Experimental Physics, Vol. 6: Capillarity and Surface Tension. Leipzig: Akademische Verlagsgesellschaft, $1928, \mathrm{xv}+458 \mathrm{p}$

19. Churaev, N. V., Derjaguin, B. V., Muller, V. M. Surface Forces. Berlin: Springer-Verlag, 1987, 440 p. 
20. Гохштейн, А. Я. Поверхностное натяжение твердых тел и адсорбция. Москва: Наука, 1976, 400 с.

21. Кикоин, А.К., Кикоин, И.К. Молекулярная физика. Москва: Наука, 1976, 480 с.

22. Ono, S., Kondo, S. Molecular Theory of Surface Tension in Liquids. In: Structure of Liquids. Encyclopaedia of Physics. BerlinHeidelberg: Springer-Verlag, 1960, Vol. 3, No. 3, p. 134-280.

23. Лабунцов, Д. А., Ягов В. В. Гидростатическое равновесие и волновые движения газожидкостных систем. Москва: Московский энергетический институт, 1977, 72 с.

24. Landau, L. D., Lifshitz, E. M. Course of Theoretical Physics, Vol. 6: Fluid Mechanics. Oxford Pergamon Press, 1987, xiii+539 p.

25. Зимон, А. Д. Адгезия жидкости и смачивания. Москва: Химия, 1974, 412 с.

26. Khomich, V. Yu., Mikolutskiy, S. I., Shmakov, V. A., Yamschikov, V. A. Model of Nanostructure Formation on Solid Surface Melted by Laser Pulse. In: Proceedings of the International Conference on Nanomaterials: Application \& Properties, 2012 , Vol. 1. No. 4, 04RES01(4 p).

27. Миколуцкий, С. И., Х Хомич, В. Ю., Ямщиков, В. А., $\quad$ Токарев, В. Н., Шмаков, В. А. Исследование процессов формирования наноструктур на поверхности материалов под действием излучения ArF-лазера. Усnехи прикладной физики, 2013, Том 1, № 4, c. 548-553.

28. Pomogailo, A. D., Kestelman, V. N. Metallopolymer Nanocomposites. Berlin-Heidelberg: Springer-Verlag, 2005 , xx+564 p.

29. Deb, P. Kinetics of Heterogeneous Solid State Processes. New York: Springer, 2014, vii+49 p.

30. Barret, P. Kinetics of Heterogeneous Processes. Paris: Gauthier-Villars, 1973, 574 p.

31. Tokarev, V. N., Shmakov, V. A., Khomich, V. Yu., Khasaya, R. R., Mikolutsky, S. I., Nebogatkin, S. V., Yamschikov, V. A. Review of Methods of Direct Laser Nanostructuring Technological Materials. In: Proceedings of the 29th International Congress on Applications of Lasers and Electrooptics, 2010, p. 1257-1265.

32. Khomich, V. Yu., Shmakov, V. A. Formation of Periodic Nanodimensional Structures on the Surface of Solids during Phase and Structural Transformations. Doklady Physics, 2012, Vol. 57, No. 9, p. 349-351.

33. Gurtin, M. E. Generalized Ginzburg-Landau and Cahn-Hilliard Equations Based on a Microforce Balance. Journal of Physics D: Nonlinear Phenomena, 1996, Vol. 92, No. 3-4, p. 178-192.

34. Hohenberg, P. C., Krekhov, A. P. An Introduction to the Ginzburg-Landau Theory of Phase Transitions and Nonequilibrium Patterns. Journal of Physics Reports, 2015, Vol. 572, p. 1-42. 